\title{
STOCHASTIC DIFFERENTIAL EQUATIONS IN A DIFFERENTIABLE MANIFOLD
}

\author{
KIYOSI ITÔ
}

The theory of stochastic differential equations in a differentiable manifold has been established by many authors from different view-points, especially by P. Lévy [2] "), F. Perrin [1], A. Kolmogoroff [1] [2] and K. Yosida [1] [2]. It is the purpose of the present paper to discuss it by making use of stochastic integrals."

In $\S 1$ we shall state some properties of stochastic integrals for the later use. We shall discuss stochastic differential equations in the $r$-dimensional Euclidean space in $\S 2$ and in a differentiable manifold in $\S 3$.

1. Some properties of stochastic integrals. Throughout this note we fix an r-dimensional Brownian motion ${ }^{3)}$ :

$$
\beta(t, \omega)=\left(\beta^{1}(t, \omega), \quad \beta^{2}(t, \omega), \ldots, \beta^{\top}(t, \omega)\right), \quad-\infty<t<\infty,
$$

$\omega(E \Omega)$ being the probability parameter with the probability law $P$ and $t$ being the time parameter. We assume that any function of $t$ and $\omega$ appearing in this note satisfies the following two conditions:

(1.2) it is measurable in $(t, \omega)$,

(1.3) the value it takes at $t=t_{0}$ is a $B$-measurable function ${ }^{4)}$ of the joint variable $\left(\beta(t, \omega), \tau \leqq t_{0}\right)$ for any $t_{0}$.

If it holds

$$
\begin{array}{r}
\xi(s, \omega)-\xi(t, \omega)=\int_{t}^{s} a(\tau, \omega) d \tau+\sum_{i=1}^{r} \int_{t}^{s} b_{i}(\tau, \omega) d \beta^{i}(\tau, \omega),{ }^{5)} \\
u \leqq s \leqq t \leqq v, \omega \in \Omega_{1}(\subseteq \Omega),
\end{array}
$$

Received March 10, 1950.

1) The numbers in [ ] denote those of the references at the end of this paper.

2) K. Itô [1], [3].

3) By an $r$-dimensional Brownian motion we understand an $r$-dimensional random process whose components are all one dimensional Brownian motion (Cf. P. Lévy [1] p. 1E6, \$52, J. L. Doob [1] Theorem 3.9) independent of each other.

4) A mapping $f$ from $R^{4}$ into $R$ is called to be B-measurable if the inverse image of any Borel subset of $R$ by $f$ is also a Borel subset of $R^{A}$, that is an element of the least completely additive class that contains all rectangular subsets of $R^{A}$.

A random variable $\zeta(\omega)$ is called to be a B-measurable function of the joint variable $(\xi \alpha(\omega), \alpha \in A)$ if and only if there exists a $B$-measurable mapping $f$ from $R^{A}$ into $R$ such that $\left.\xi(\omega)=f\left(\xi_{\alpha} \alpha\right), \alpha \in A\right)$ for every $\omega$. Cf. K. Itô [3] $\$ 1$.

5) The sense of this integral is to be understcod as a stochastic integral introduced by the author. Cf. K. Itô [1], [3] $§ 7, \S 8$. 
then we shall express this relation in the differential form as follows:

$$
d \xi(t, \omega)=a(t, \omega) d t+\sum_{j=1}^{r} b_{j}(t, \omega) d \beta^{j}(t, \omega), \quad u \leqq t \leqq v, \omega \in \Omega_{1} .
$$

TheOREM 1.1. If

(1.6) $d \xi^{i}(t, \omega)=a^{i}(t, \omega) d t+\sum_{j=1}^{r} b_{j}^{i}(t, \omega) d \beta^{j}(t, \omega), i=1,2, \ldots, m, u \leqq t \leqq v$, $\omega \in \Omega_{1}$, and if

(1.7) $\quad \eta(t, \omega)=f(\xi(t, \omega)), \quad \xi(t, \omega)=\left(\xi^{1}(t, \omega), \xi^{2}(t, \omega), \ldots, \xi^{m}(t, \omega)\right)$,

$f$ being a real-valued function of $C_{2}$-class ${ }^{6)}$ defined on an open subset of $R^{m}$ which contains all the points $\xi(t, \omega), u \leqq t \leqq v, \omega \in \Omega_{1}$, then we have

$$
\begin{aligned}
d \eta(t, \omega) & =\left\{\sum_{i} f_{i}(\xi(t, \omega)) a^{i}(t, \omega)+\frac{1}{2} \sum_{i j k} f_{i j}(\xi(t, \omega)) b_{k}^{i}(t, \omega) b_{k}^{j}(t, \omega)\right\} d t \\
& +\sum_{j}\left(\sum_{i} f_{i}(\xi(t, \omega)) b_{j}^{i}(t, \omega)\right) d \beta^{j}(t, \omega),
\end{aligned}
$$

where

$$
f_{i}\left(x^{1}, \ldots, x^{m}\right)=\frac{\partial f}{\partial x^{i}}\left(x^{1}, \ldots, x^{m}\right), f_{i j}\left(x^{1}, \ldots, x^{m}\right)=\frac{\partial^{2} f}{\partial x^{i} \partial x^{j}}\left(x^{1}, \ldots, x^{m}\right)
$$

We shall here mention only the outline of the proof. ${ }^{7)}$ First we shall state a lemma.

LEMMA.

$$
\begin{aligned}
& \int_{s}^{t} b(\tau, \omega) d \beta^{i}(\tau, \omega) \int_{s}^{t} c(\tau, \omega) d \beta^{j}(\tau, \omega) \\
= & \delta^{i j} \int_{s}^{t} b(\tau, \omega) c(\tau, \omega) d \tau+\int_{s}^{t} b(\tau, \omega) \int_{s}^{\tau} c(\sigma, \omega) d \beta^{j}(\sigma, \omega) d \beta^{i}(\tau, \omega) \\
+ & \int_{s}^{t} c(\sigma, \omega) \int_{s}^{\sigma} b(\tau, \omega) d \beta^{i}(\tau, \omega) d \beta^{j}(\sigma, \omega) \quad\left(\delta^{i j}=\text { Kronecker's delta }\right) .
\end{aligned}
$$

We can prove this lemma first by considering the special case that both $b(\tau, \omega)$ and $c(\tau, \omega)$ are uniformily stepwise ${ }^{s)}$ in $(s, t)$ and next by taking the limit in the general case.

In order to prove (1.8) we need only to compute the following expression:

$$
\begin{aligned}
& \eta(s, \omega)-\eta(t, \omega)=\sum_{\nu=1}^{n}\left(\eta\left(t_{\nu}^{(n)}, \omega\right)-\eta\left(t_{\nu-1}^{(n)}, \omega\right)\right), t_{\nu}^{(n)}=t+\frac{\nu}{n}(s-t), \\
& =\sum_{\nu i} f_{i}\left(\xi\left(t_{\nu-1}^{(n)}, \omega\right)\right)\left(\xi^{i}\left(t_{\nu}^{(n)}, \omega\right)-\xi^{i}\left(t_{\nu-1}^{(n)}, \omega\right)\right) \\
& +\frac{1}{2} \sum_{\nu i j}\left(f_{i j}\left(\xi\left(t_{\nu-1}^{(n)}, \omega\right)\right)+\varepsilon_{i j \nu}(\omega)\right)\left(\xi^{i}\left(t_{\nu}^{(n)}, \omega\right)-\xi^{i}\left(t_{\nu-1}^{(n)}, \omega\right)\right)\left(\xi^{j}\left(t_{\nu}^{(n)}, \omega\right)-\xi^{j}\left(t_{\nu-1}^{(n)}, \omega\right)\right)
\end{aligned}
$$

6) A function is called to be of $C_{2}$-class if its partial derivatives of the second order are all continuous.

7) The author will publish the proof in details in another note.

8) $b(\tau, \omega)$ is called to be uniformly stepwise in $(s, t)$ if there exist a division of the interval $(s, t): s=t_{0}<t_{1}<\ldots<t_{n}=t$ independent of $\omega$ such that $b(\tau, \omega) b\left(t_{i-1}, \omega\right), t_{i-1}$ $\leqq \tau \leqq t_{i}, i=1,2, \ldots, n$. Cf. K. Itô [1], [3] $\$ 7$. 
by making use of the above lemma and to take the limit as $n \rightarrow \infty$.

TheOREM 1.2. The functions $a(t, \omega), b_{i}(t, \omega), i=1,2, \ldots, r$, are uniquely determined by $\xi(t, \omega)$ in the sense that

implies

$$
\begin{aligned}
d \xi(t, \omega) & =a(t, \omega) d t+\sum_{i} b_{i}(t, \omega) d \beta^{i}(t, \omega) \\
& =a(t, \omega) d t+\sum_{i} \widetilde{b}_{i}(t, \omega) d \beta^{i}(t, \omega), \quad u \leqq t \leqq v, \quad \omega \in \Omega_{1},
\end{aligned}
$$

$$
a(t, \omega)=\tilde{a}(t, \omega), \quad b_{i}(t, \omega)=\tilde{b}_{i}(t, \omega), \quad i=1,2, \ldots, r,
$$

for almost all $(t, \omega), u \leqq t \leqq v, \omega \in \Omega_{1}$.

Proof. It suffices to show that

implies

$$
\xi(t, \omega) \equiv 0, \quad u \leqq t \leqq v, \quad \omega \in \Omega_{1},
$$

$$
a(t, \omega)=0, \quad b_{i}(t, \omega)=0, \quad i=1,2, \ldots, r,
$$

for almost all $(i, \omega), u \leqq t \leqq v, \omega \in \Omega_{1}$.

By Theorem 1 we have

$$
\begin{aligned}
(\xi(s, \omega)-\xi(t, \omega))^{2}= & \int_{s}^{t}\left\{2(\xi(\tau, \omega)-\xi(s, \omega)) a(\tau, \omega)+\sum_{i} b_{i}(\tau, \omega)^{2}\right\} d \tau \\
& +\sum_{i} \int_{s}^{t} 2(\xi(\tau, \omega)-\xi(s, \omega)) b_{i}(\tau, \omega) d \beta^{i}(\tau, \omega),
\end{aligned}
$$

from which it follows that

$$
\sum_{i=1}^{n}\left(\xi\left(u+\frac{\nu}{n}(v-u), \omega\right)-\xi\left(u+\frac{\nu-1}{n}(v-u),(v)\right)^{2} \rightarrow \int_{u}^{v} \sum_{i} b_{i}(\tau, \omega)^{2} d \tau\right.
$$

in probability. But the left side is always equal to 0 by the assumption and so we obtain

$$
\int_{u}^{v} \sum_{i} b_{i}(\tau, \omega)^{2} d \tau=0
$$

for almost all $\omega$ in $\Omega_{1}$, which completes the proof.

We have already obtained the following inequality ${ }^{\text {) }}$

$$
\begin{aligned}
\left.\operatorname{Pr} \sup _{u \leqq t \leqq v}\left|\int_{u}^{t} b(\tau, \omega) d \beta^{j}(\tau, \omega)\right| \equiv \alpha\right\} & \leqq \frac{1}{\alpha^{2}} \int_{u}^{v} E\left(b(\tau, \omega)^{2}\right) d t \\
& =O(v-u) \quad(\text { as } v \rightarrow u) .
\end{aligned}
$$

The following theorem gives us a more precise evaluation in the case that $b(t, \omega)$ is uniformly bounded.

Theorem 1.3. In the case:

$$
|b(t, \omega)| \leqq K \quad(u \leqq t \leqq v, \omega \in \Omega), \quad 0 \leqq v-u<\alpha^{2} /\left(2 K^{2}\right),
$$

we have

$$
\begin{aligned}
P_{r}\left\{\sup _{u \leqq t \leqq v}\left|\int_{u}^{t} b(\tau, \omega) d \beta^{j}(\tau, \omega)\right| \geqslant \alpha\right\} & \leqq \frac{8 K^{4}(v-u)^{2}}{\alpha^{4}} \\
& =O(v-u)^{2} \quad(\text { as } v \rightarrow u) .
\end{aligned}
$$

9) Cf. K. Itô [1] Th. 8, [3]. $P_{\boldsymbol{r}}(\mathfrak{A}(\omega))=P(\{\omega ; \mathfrak{A}(\omega)\}), E(\xi(\omega))=\int_{\Omega^{\xi}(\omega) P(d \omega)}$. 
Proof. By Theorem 1.1 we have

$$
\left(\int_{u}^{v} b(t, \omega) d \beta^{j}(t, \omega)\right)^{2}=\int_{u}^{v} b(t, \omega)^{2} d t+2 \int_{u}^{v} b(t, \omega) \int_{u}^{t} b(\tau, \omega) d \beta^{j}(\tau, \omega) d \beta^{j}(t, \omega) .
$$

But (1.10) implies

$$
\int_{u}^{v} b(t, \omega)^{2} d t \leqq \frac{\alpha^{2}}{2} .
$$

Therefore the left side of (1.11) is less than

$$
\begin{gathered}
\operatorname{Pr}\left\{\sup _{u \leqq s \leqq v}\left|\int_{u}^{s} b(t, \omega) \int_{\sigma}^{t} b(\tau, \omega) d \beta^{j}(\tau, \omega) d \beta^{j}(t, \omega)\right| \geqq \frac{\alpha^{2}}{4}\right\} \\
\leqq\left(\frac{4}{\alpha^{2}}\right)^{2} \int_{u}^{v} E\left\{\left(b(t, \omega) \int_{u}^{t} b(\tau, \omega) d \beta^{j}(\tau, \omega)\right)^{2}\right\} d t \leqq\left(\frac{4}{\alpha^{2}}\right)^{2} K^{4} \frac{(v-u)^{2}}{2}=\frac{8 K^{4}(v-u)^{2}}{\alpha^{4}}
\end{gathered}
$$

on account of (1.9).

2. Stochastic differential equation on the $r$-dimensional Euclidean space.

In this paragraph we shall treat a stochastic differential equation:

$$
\begin{gathered}
d \xi^{i}(t, \omega)=a^{i}(t, \xi(t, \omega)) d t+\sum_{j} b_{j}{ }^{i}(t, \xi(t, \omega)) d \beta^{j}(t, \omega), \\
\xi(t, \omega)=\left(\xi^{1}(t, \omega), \ldots, \xi^{r}(t, \omega)\right),,^{10)} \quad i=1,2, \ldots, r, \quad u \leqq t \leqq v,
\end{gathered}
$$

with the initial condition

$$
\xi^{i}(u, \omega)=c^{i}(\omega), i=1,2, \ldots, r,
$$

where $c^{i}(\omega), i=1,2, \ldots, r$ are $B$-measurable functions of $(\beta(\tau, \omega), \tau \leqq u)$. This stochastic differential equation is equivalent to a stochastic integral equation :

$$
\xi^{i}(t, \omega)=c^{i}(\omega)+\int_{u}^{t} a^{i}(\tau, \xi(\tau, \omega)) d \tau+\sum_{j} \int_{u}^{t} b_{j}{ }^{i}(\tau, \xi(\tau, \omega)) d \beta^{j}(\tau, \omega), \quad u \leqq t \leqq v .
$$

THEOREM 2.1. Under the following four assumptions ${ }^{11}$ :

where

$$
\begin{gathered}
\sum_{i}\left|a^{i}(t, x)-a^{i}(t, y)\right|^{2} \leqq A\|x-y\|^{2}, \\
\sum_{i j}\left|b_{j}{ }^{i}(t, x)-b_{j}{ }^{i}(t, x)\right|^{2} \leqq B\|x-y\|^{2},
\end{gathered}
$$

$$
\begin{gathered}
\|x-y\|^{2}=\sum_{i}\left|x^{i}-y^{i}\right|^{2}, \\
a^{i}(t, x), b_{j}{ }^{i}(t, x) \text { are all continuous in } t \text { for any } x . \\
\sum_{i}\left|a^{i}(t, x)\right|^{2} \leqq A_{1}, \quad \sum_{i j}\left|b_{j}{ }^{i}(t, x)\right|^{2} \leqq B_{1}, \\
E\left(\left|c^{i}(\omega)\right|^{2}\right) \leqq C, \quad i=1,2, \ldots, r,
\end{gathered}
$$

$\left(A, B, A_{1}, B_{1}, C\right.$ being constants independent of $(t, x)$ ), there exists one and only one solution of the equation (2.3).

19) The $i$-component of $r$-dimensional vectors $x, y, c(\omega), \xi(t, \omega), \eta(t, \omega)$ etc. are denoted by $x^{i}, y^{i}, c^{i}(\omega), \xi^{i}(t, \omega), \eta^{i}(t, \omega)$ etc. respectively.

11) It is possible to show this theorem without any use of the assumption (2.6), (2.7) by the method the author has used in one-dimensional case (K. Itô [1], [3] Th. 11) but it is unnecessary for our present purpose to do so. 
Proof of the existence. We shall make use of the successive approximation method to find a solution of (2.3). We define $\xi_{n}(t, \omega), n=1.2, \ldots$, recurively as follows :

$$
\begin{gathered}
\xi_{0}{ }^{i}(t, \omega)=c^{i}((1)), \quad i=1,2, \ldots, r \\
\xi_{n}{ }^{i}(t, \omega)=c^{i}(\omega)+\int_{u}^{t} a^{i}\left(\tau, \xi_{n-1}(\tau, \omega)\right) d \tau+\sum_{j} \int_{u}^{t} b_{j}^{i}\left(\tau, \xi_{n-1}(\tau, \omega)\right) d \beta^{j}(\tau, \omega) .
\end{gathered}
$$

Then we have

$$
\begin{gathered}
\sum_{i}\left|\xi_{1}^{i}(t, \omega)-\xi_{0}^{i}(t, \omega)\right|^{2}=\sum_{i}\left|\int_{u}^{t} a^{i}\left(\tau, \xi_{0}(\tau, \omega)\right) d \tau+\sum_{j} \int_{u}^{t} b_{j}{ }^{i}\left(\tau, \xi_{0}(\tau, \omega)\right) d \beta^{j}(\tau, \omega)\right|^{2} \\
\leqq(r+1) \sum_{i}\left\{\left|\int_{u}^{t} a^{i}\left(\tau, \xi_{0}(\tau, \omega)\right) d \tau\right|^{2}+\sum_{j}\left|\int_{u}^{t} b_{j}{ }^{i}\left(\tau, \xi_{0}(\tau, \omega)\right) d \beta^{j}(\tau, \omega)\right|^{2}\right\},
\end{gathered}
$$

(2.10) $E\left(\left\|\xi_{1}(t, \omega)-\xi_{0}(t, \omega)\right\|^{2}\right)$

$$
\begin{aligned}
& \leqq(r+1)\left\{\sum_{i}(t-u) \int_{u}^{t} E\left(a^{i}\left(\tau, \xi_{0}(\tau, \omega)\right)^{2}\right) d \tau+\sum_{i j} \int_{u}^{t} E\left(b_{j}{ }^{i}\left(\tau, \xi_{0}(\tau, \omega)\right)^{2}\right) d \tau\right\} \\
& \leqq(r+1)\left\{(v-u) \int_{u}^{t} E\left(\sum_{i} a^{i}\left(\left(\tau, \xi_{0}(\tau, \omega)\right)^{2}\right) d \tau+\int_{u}^{t} E\left(\sum_{i j} b_{j}^{i}\left(\tau, \xi_{0}(\tau, \omega)\right)^{2}\right) d \tau\right\}\right. \\
& \leqq(r+1)\left\{(v-u) A_{1}+B_{1}\right\}(t-u),
\end{aligned}
$$

in making use of (2.6). In the same manner we obtain

$$
\begin{aligned}
& E\left(\left\|\xi_{n}(t, \omega)-\xi_{n-1}(t, \omega)\right\|^{2}\right) \\
\leqq & (r+1)\{(v-u) A+B\} \int_{u}^{t} E\left\{\left\|\xi_{n-1}(\tau, \omega)-\xi_{n-2}(\tau, \omega)\right\|^{2}\right\} d \tau
\end{aligned}
$$

by virtue of (2.4). From (2.10) and (2.11) it follows that

$$
E\left(\left\|\xi_{n}(t, \omega)-\xi_{n-1}(t, \omega)\right\|^{2}\right) \leqq \frac{K^{n}(t-u)^{n}}{n !} \leqq \frac{K^{n}(v-u)^{n}}{n !},
$$

$K$ being a constant determined by $A_{1}, B_{1}, A, B, u$ and $v$.

Since

$$
\sum_{n=1}^{\infty} \sqrt[3]{\frac{K^{n}(v-u)^{n}}{n !}}<\infty
$$

we see, by Borel-Cantelli's theorem, that there exists a function $\xi(t, \omega)$ such that

$$
E\left(\left\|\xi(t, \omega)-\xi_{n}(t, \omega)\right\|^{2}\right) \rightarrow 0 \text { for each } t .
$$

$\xi^{i}(t, \omega), i=1,2, \ldots, r$, are clearly $B$-measurable functions of $(\beta(\tau, \omega), \tau \leqq t)$ for each $t$. But we have further

$$
\int_{u}^{v} E\left(\left\|\xi_{m}(t, \omega)-\xi_{n}(t, \omega)\right\|^{2}\right) d t \rightarrow 0 \quad(m, n \rightarrow \infty),
$$

from which we see that $\xi^{i}(t, \omega), i=1,2, \ldots, r$, are all measurable in $(t, \omega)$.

Taking the limit of (2.9) as $n \rightarrow \infty$, we see that $\xi(t, \omega)$ satisfies (2.3).

Proof of the uniqueness. Let $\xi(t, \omega)$ and $\tilde{\xi}(t, \omega)$ satisfies $(2.3)$. In the same manner as (2.12) we have 


$$
E\left(\|\left(\xi(t, \omega)-\tilde{\xi}(t, \omega) \|^{2}\right) \leqq \frac{K^{n}(v-u)^{n}}{n !} \rightarrow 0,\right.
$$

which completes the proof.

Theorem 2.2. Assume (2.4), (2.5), (2.6), (2.7) and

$$
a^{i}(t, x), \quad b_{j}^{i}(t, x) \equiv 0 \text { for } x \neq U
$$

and

$$
c(\omega) \in U,
$$

$U$ being an open subset of $R^{r}$. Then the solution $\xi(t, \omega)$ of $(2.3)$ satisfies

$$
\xi(t, \omega) \in U, u \leqq t \leqq v,
$$

for almost all $\omega$.

Proof. It suffices to prove that the totality $\Omega_{1}$ of $\omega$ for which $\xi(t, \omega) \notin U$ for some $t$ has $P$-measure 0 . Assume that $P\left(\Omega_{1}\right)>0$. Since $\xi(t, \omega)$ is continuous in $t$ for each $\omega$, there exist $t$ and $s(u \leqq t \leqq s \leqq v)$ such that

$$
\xi(\tau, \omega) \notin U \text { for } t \leqq \tau \leqq s, \quad \xi(t, \omega) \neq \xi(s, \omega)
$$

on an $\omega$-set $\Omega_{2}\left(\subseteq \Omega_{1}\right)$ with positive $P$-measure. From (2.13), (2.14), and (2.16) it follows that

$$
a^{i}(\tau, \xi(\tau, \omega))=0, \quad b_{j}^{i}(\tau, \xi(\tau, \omega))=0, \quad s \leqq \tau \leqq t, \quad \omega \in \Omega_{2},
$$

and so we have

$$
\xi^{i}(s, \omega)=\xi^{i}(t, \omega)+\int_{s}^{t} a^{i}(\tau, \xi(\tau, \omega)) d \tau+\int_{s}^{t} b_{j}{ }^{i}(\tau, \xi(\tau, \omega)) d \beta^{j}(\tau, \omega)=\xi^{i}(t, \omega) .
$$

almost everywhere on $\Omega_{\mathrm{s}}$, which contradicts with (2.16).

\section{Stochastic differential equation in a differentiable manifold.}

Given any $r$-dimensional differentiable manifold of $C_{2}$-class ${ }^{12)} M$. By a continuous random motion in $M$ we understand an $M$-valued function $\pi(t, \omega)$ of $t$ and $\omega$ which is measurable in $\omega$ for each $t$ and continuous in $t$ for each $\omega$. Hereafter we assume that

$$
\pi(t, \omega) \text { is a } B \text {-measurable function of }(\beta(\tau, \omega), \tau \leqq t) .
$$

Let $\xi(t, \omega) \equiv\left(\xi^{1}(t, \omega), \xi^{2}(t, \omega), \ldots, \xi^{r}(t, \omega)\right)$ be any local coordinate of $\pi(t, \omega)$. If. we define $\xi(t, \omega) \equiv 0$ in case $\pi(t, \omega)$ is outsides of the coordinate neighbourhood, $\xi^{i}(t, \omega)$ proves to be a $B$-measurable function of $\beta(\tau, \omega), \tau \leqq t$, on account of (3.1). We can easily see that $\xi^{i}(t, \omega)$ is measurable in $(t, \omega)$.

We shall consider a stochastic differential equation:

12) By definition an $r$-dimensional differentiable manifold of $C_{z}$-class is a Hausdorff space with the second countability axiom and with coordinate neighbourhoods, each homeomorphic to the interior of a sphere of $r$-dimensional Euclidean space and such that the coordinate relationships between the coordinates of the two intersecting neighbourhoods are of $C_{2}$-class. 
which means that

$$
d \hat{\xi}^{i}(t, \omega)=a^{i}(t, \xi(t, \omega)) d t+\sum_{j} b_{j}^{i}(t, \xi(t, \omega)) d \beta^{j}(t, \omega),
$$

$$
\xi^{i}(t, \omega)-\xi^{i}(s, \omega)=\int_{s}^{t} a^{i}(\tau, \xi(\tau, \omega)) d \tau+\sum_{j} \int_{s}^{t} b_{j}^{i}(\tau, \xi(\tau, \omega)) d \beta^{j}(\tau, \omega)
$$

for any $\omega$ such that $\pi(\xi, \omega)$ is contained in the coordinate neighbourhood for $s \leqq \tau \leqq t$.

The functions $a^{i}(t, x)$ and $b_{j}{ }^{i}(t, x),\left(x=\left(x^{1}, x^{2}, \ldots, x^{r}\right)\right)$ depend upon the special choice of the local coordinate. We assume that they are transformed between two local coordinates $x$ and $\bar{x}$ in the following manner:

$$
\left\{\begin{aligned}
\bar{a}^{i}(t, \bar{x}) & =\sum_{k} \frac{\partial \bar{x}^{i}}{\partial x^{k}} a^{k}(t, x)+\frac{1}{2} \sum_{j k l} \frac{\partial^{2} \bar{x}^{i}}{\partial x^{k} \partial x^{l}} b_{j}{ }^{k}(t, x) b_{j}{ }^{l}(t, x), \\
\bar{b}_{j}{ }^{i}(t, \bar{x}) & =\sum_{k} \frac{\partial \bar{x}^{i}}{\partial x^{k}} b_{j}{ }^{k}(t, x) .
\end{aligned}\right.
$$

Now we shall explain the reason why we choose such a transformation law. Let $\bar{x}=\left(\bar{x}^{1}, \bar{x}^{2}, \ldots, \bar{x}^{r}\right)$ and $x=\left(x^{1}, x^{2}, \ldots, x^{r}\right)$ be two local coordinates defined on a neighbourhood $U$ in $M$ such that

$$
\bar{x}^{i}=f^{i}\left(x^{1}, x^{2}, \ldots, x^{r}\right), i=1,2, \ldots, r,
$$

and let $\xi(t, \omega)(\bar{\xi}(t, \omega))$ denote the $x(\bar{x})$-coordinate of $\pi(t, \omega)$. Then we have (3.2) and

$$
\begin{gathered}
d \bar{\xi}^{i}(t, \omega)=\bar{a}^{i}(t, \bar{\xi}(t, \omega)) d t+\sum_{j} \bar{b}_{j}^{i}(t, \bar{\xi}(t, \omega)) d \beta^{j}(t, \omega), \\
\bar{\xi}^{i}(t, \omega)=f^{i}\left(\xi^{1}(t, \omega), \quad \xi^{2}(t, \omega), \ldots, \xi^{r}(t, \omega)\right), \quad i=1,2, \ldots, r .
\end{gathered}
$$

In making use of Theorem 1.1 we deduce from (3.2) and (3.5)

$$
\begin{aligned}
d \bar{\xi}^{i}(t, \omega)=\left\{\sum_{k} f_{k}{ }^{i}(\xi(t, \omega)) a^{k}(t, \xi(t, \omega))\right. & \\
& \left.+\frac{1}{2} \sum_{k l j} f_{k l}^{i}(\xi(t, \omega)) b_{j}{ }^{k}(t, \xi(t, \omega)) b_{j}{ }^{l}(t, \xi(t, \omega))\right\} d t \\
& +\sum_{j} \sum_{k} f_{k}{ }^{i}(\xi(t, \omega)\} b_{j}{ }^{k}(t, \xi(t, \omega)) d \beta^{j}(t, \omega),
\end{aligned}
$$

whenever $\pi(t, \omega)$ is contained in $U$. By Theorem 1.2 it follows from $\left(3.2^{\prime}\right)$ and (3.6) that

$$
\begin{aligned}
\bar{a}^{i}(t, \xi(t, \omega))= & \sum_{k} f_{k}{ }^{i}(\xi(t, \omega)) a^{k}(t, \xi(t, \omega)) \\
& \quad+\frac{1}{2} \sum_{k l j} f_{k l}^{i}(\xi(t, \omega)) b_{j}^{k}(t, \xi(t, \omega)) b_{j}{ }^{l}(t, \xi(t, \omega)), \\
\bar{b}_{j}{ }^{i}(t, \xi(t, \omega))= & \sum_{k} f_{k}{ }^{i}(\xi(t, \omega)) b_{j}{ }^{k}(t, \xi(t, \omega)) .
\end{aligned}
$$

In order to express a sufficient condition for the equation (3.2) to have a unique solution, we define the boundedness of $a^{i}(t, x)$ and $b_{j}{ }^{i}(t, x)$ as follows.

DEFINITION. By a canonical coordinate around $p$ we understand any local coordinate which maps a neighbourhood of $p$ onto the interior of the unit sphere 
in the $r$-dimensional Euclidean space $R^{r}$ and especially transforms $p$ to the centre of the sphere.

By a canonical coordinate system on $M$ we understand a collection of canonical coordinates such that, for any point of $M$, there exists a canonical coordinate around the point in the collection.

$a^{i}(t, x), b_{j}{ }^{i}(t, x), i, j=1,2, \ldots, r$ are called to be bounded if and only if there exists a canonical coordinate system and a constant $K$ such that we have always

$$
|a(t, x)|,|b(t, x)|<K, i, j=1,2, \ldots, r, u \leqq t \leqq v, x \in S,
$$

for any coordinate of the system.

THEOREM 3.1. We assume that $a^{i}(t, x), b_{j}{ }^{i}(t, x), i, j=1,2, \ldots, r, 0 \leqq t \leqq 1$, are all

$$
\text { bounded in the sense of the above definition, }
$$$$
\text { continuous in } t \text { for each } x \text {, }
$$

and

(3.10) of $C_{1}$-class in $x$ for each $t$.

Then there exists one and only one solution of the stochastic differential equation (3.2) with the initial condition:

$$
\pi(u, \omega)=p(\omega),
$$

where $p(\omega)$ is an M-valued B-measurable function of $(\beta(\tau, \omega), \tau \leqq t)$.

Proof of the existence. We shall denote by $\left\{x_{p}=\cdot\left(x_{p}{ }^{1} \ldots x_{p}^{r}\right): p \in M\right\}$ a canonical coordinate system which satisfies (3.7) and by $S\left(S_{1}, S_{2}\right)$ the interior of the sphere with the centre at the origin 0 and with the redius $1(1 / 3,2 / 3)$, and by $U_{p}\left(U_{p}^{\prime}\right)$ the totality of all the points of $M$ whose $x_{p}$-coordinate lies in $S\left(S_{1}\right)$. Since $M$ satisfies the second countability axiom, we obtain a sequence in $M:\left\{p_{n}\right\}$ for which $\left\{U_{p_{n}}{ }^{\prime}\right\}$ covers the whole space $M$. We denote $x_{p_{n}}, U_{p_{n}}$ and $U_{p_{n}{ }^{\prime}}$ respectively by $x_{n} \equiv\left(x_{n}{ }^{1}, \ldots, x_{n}{ }^{r}\right), U_{n}$ and $U_{n}{ }^{\prime}$. We define $V_{n}$ by

$$
V_{n}=U_{n^{\prime}}-\bigcup_{i=1}^{n-1} U_{i}^{\prime} \quad(n \geqq 2), \quad V_{1}=U_{1}^{\prime} .
$$

It is clear that

$$
M=\sum_{n=1}^{\infty} V_{n} \cdot{ }^{13)}
$$

Now we shall define a random motion $\pi_{m}(t, \omega)$ which satisfies the equation (3.2) on an $\omega$-set $\left(\Omega^{\langle m i}\right)$ whose $P$-measure tends to 1 as $m \rightarrow \infty$.

We define a function $\lambda(x)$ on $R^{r}$ by

13) In this paper we denote the sum of disjoint sets by $\Sigma$. 


$$
\lambda(x)= \begin{cases}1 & (\|x\| \leqq 2 / 3) \\ 0 & (\|x\| \equiv 5 / 6) \\ 5-6\|x\| & (2 / 3<\|x\|<5 / 6)\end{cases}
$$

and $\tilde{a}^{i}(i, x)$ and $\widetilde{b}_{j}^{i}(t, x)$ by

$$
\widetilde{a}^{i}(t, x)=a(t, x) \lambda(x), \quad \widetilde{b}_{j}^{i}(t, x)=b_{j}^{i}(t, x) \lambda(x) .
$$

Then $\widetilde{a}^{i}(t, x)$ and $\widetilde{b}_{j}{ }^{i}(t, x)$ satisfies the assumptions in Theorem 2.1 and

$$
\widetilde{a}^{i}(t, x)=0, \widetilde{b}_{j}^{i}(t, x)=0, \quad i, j=1,2, \ldots, r, \quad x \notin S .
$$

We devide the time-interval $(u, v)$ into $m$ equal subintervals with

$$
u=u_{0}<u_{1}<u_{2}<\ldots<u_{m}=v .
$$

We define $C_{n 1}(\omega)=\left(C_{n 1}^{1}(\omega), \ldots, C_{n 1}^{r}(\omega)\right)$ by

$$
C_{n \mathrm{1}}(\omega)= \begin{cases}\text { the } x_{n} \text {-coordinate of } p(\omega) & \text { if } p(\omega) \in V_{n} \\ 0 \equiv(0,0, \ldots, 0) & \text { if } p(\omega) \notin V_{n},\end{cases}
$$

and consider a stochastic integral equation:

$$
\begin{gathered}
\hat{\varsigma}^{i}(t, \omega)=C_{n 1}^{i}(\omega)+\int_{u_{0}}^{t} \tilde{a}^{i}\left(\tau, \xi_{n}(\tau, \omega)\right) d \tau+\sum_{j} \int_{u_{0}}^{t} \widetilde{b}_{j n}^{i}\left(\tau, \xi_{n}(\tau, \omega)\right) d \beta^{j}(\tau,(1), \\
i=1,2, \ldots, \boldsymbol{r}, \quad u_{0} \leqq t \leqq u_{1} .
\end{gathered}
$$

By Theorem 2.1 and Theorem 2.2 we can see that this equation has a unique solution such that

$$
\xi_{n}(t, \omega) \in S, \quad u_{0} \leqq t \leqq u_{1},
$$

for almost all $\omega$.

We define $\pi_{m}(t, \omega), u_{0} \leqq t \leqq u_{1}$, in the following way:

(3.11) $\pi_{m}(t, \omega)=$ the point in $M$ with the $x_{n}$-coordinate $\xi_{n}(t, \omega)$ when $p(\omega) \in V_{n}$; $\pi_{m}(t, \omega), u_{0} \leqq t \leqq u_{1}$, is clearly determined up to $P$-measure 0 .

Next we consider a stochastic differential equation:

$$
\begin{gathered}
\xi_{n}^{i}(t, \omega)=C_{n 1}^{i}(\omega)+\int_{u_{1}}^{t} \widetilde{a}_{n}^{i}\left(\tau, \xi_{n}(\tau, \omega)\right) d \tau+\sum_{j} \int_{u_{1}}^{t} \widetilde{b}_{j n}^{i}\left(\tau, \xi_{n}(\tau, \omega)\right) d \beta^{j}(\tau, \omega) ; \\
i=1,2, \ldots, r, u_{1} \leqq t \leqq u_{2},
\end{gathered}
$$

where

$$
C_{n:}((1))= \begin{cases}\text { the } x_{n} \text {-coordinate of } \pi_{n}(u, \omega) & \text { if } \pi_{m}\left(u_{1}, \omega\right) \in V_{n} \\ 0 \equiv(0,0, \ldots, 0) & \text { if } \pi_{m}\left(u_{1}, \omega\right) \notin V_{n} .\end{cases}
$$

In making use of Theorem 2.1 and Theorem 2.2 again we see that this equation has a unique solution such that

$$
\xi_{n}(t, \omega) \in S, \quad u_{1} \leqq t \leqq u_{\Omega},
$$

for almost all $\omega_{\text {。 }}$ We define $\pi_{m}(t, \omega), u_{1} \leqq t \leqq u_{2}$ by (3.11) again. By continuing 
this procedure we define $\pi_{m}(t, \omega), u \leqq t \leqq v$. Now we define $\Omega_{n l}^{k}$ by

$$
\Omega_{n l}^{k}=\left\{\omega ; \pi_{m}\left(u_{k-1}, \omega\right) \in V_{n}, \pi_{m}\left(u_{k}, \omega\right) \in V_{l}, \xi_{n}(\tau, \omega) \in S_{2} \text { for } u_{k-1} \leqq \tau \leqq u_{k}\right\},
$$

and put

$$
\Omega^{(m)}=\sum_{n_{0} n_{1} \ldots n_{m}} \Omega_{n_{0} n_{1}}^{1} \Omega_{n_{1} n_{2}}^{2} \ldots \Omega_{n_{m-1} n_{m}}^{m} .
$$

We shall prove that

$$
\pi_{m}(t, \omega) \text { satisfies (3.2) almost everywhere on } \Omega^{(m)}
$$
and that

$$
P\left(\Omega^{(m)}\right) \rightarrow 1 \text { as } m \rightarrow \infty .
$$

Let $x$ be any local coordinate defined on $U$, and $\pi_{m}(t, \omega)$ lie in $U$ for $t_{1} \leqq \tau$ $\leqq t_{2}$ and $\omega \in \Omega^{*}\left(\subseteq \Omega^{(m)}\right)$. In order to show (3.12) it is sufficient to prove that the $x$-coordinate $\xi(t, \omega)$ of $\pi_{m}(t, \omega)$ satisfies

$$
d \xi^{i}(t, \omega)=a^{i}(t, \xi(t, \omega)) d t+\sum_{j} b_{j}^{i}(t, \xi(t, \omega)) d \beta^{j}(t, \omega), t_{1} \leqq t \leqq t_{2}, \omega \in \Omega^{*}
$$

In case $\omega \in \Omega_{n_{0} n_{1}}^{1} \Omega_{n_{1} n_{2}}^{2} \ldots \Omega_{n_{m-1} n_{m}}^{m} \Omega^{*}$ we have

$$
\begin{aligned}
& d \xi_{n_{k-1}}^{i}(t, \omega)=\widetilde{a}_{n_{k-1}}^{i}\left(t, \xi_{n_{k-1}}(t, \omega)\right) d t+\sum_{j} \tilde{b}_{n_{k-1}}^{i} j\left(t, \xi_{n_{k-1}}(t, \omega)\right) d \beta^{j}(t, \omega), \\
& u_{k-1} \leqq t \leqq u_{k},
\end{aligned}
$$

and so

$$
\begin{aligned}
& d \xi_{n_{k-1}}^{i}(t, \omega)=a_{n_{k-1}}^{i}\left(t, \xi_{n_{k-1}}(t, \omega)\right) d t+\sum_{j} b_{n_{k-1} j}^{i}\left(t, \xi_{n_{k-1}}(t, \omega)\right) d \beta^{j}(t, \omega), \\
& u_{k-1} \leqq t \leqq u_{k},
\end{aligned}
$$

since $\xi_{n_{k-1}}(t, \omega) \in S_{2}$ by the definition of $\Omega_{n_{k-1} n_{k}}$.

By Theorem 1.1 and the transformation law (3.3) we can deduce (3.14) from (3.15), which proves (3.12).

In order to prove (3.3) it suffices to show

$$
\begin{array}{r}
P\left\{\Omega_{n_{0} n_{1}}^{1} \ldots \Omega_{n_{k-1} n_{k}}^{k} \widetilde{\Omega}_{n_{k}}\right\} \supseteqq\left(1-G(1 / m)^{2}\right) P\left(\Omega_{n_{0} n_{1}}^{1} \ldots \Omega_{n_{k-1} n_{k}}^{k}\right), \\
k=1,2, \ldots,
\end{array}
$$

where $\widetilde{\Omega}_{n_{k}}=\sum_{n=1}^{\infty} \Omega_{n_{h} n}$ and $G$ is a constant which depends neither on $m$ nor on $k$ but only on $K, u$ and $v$; in fact it follows from (3.16) that

$$
\begin{array}{r}
P\left(\Omega^{m}\right) \geqq\left(1-G(1 / m)^{2}\right) \sum P\left(\Omega_{n_{0} n_{1}}^{1} \Omega_{n_{1} n_{2}}^{2} \ldots \Omega_{n_{m^{\prime}-2} n_{m-1}}^{m-1}\right) \geqq\left(1-G(1 / m)^{2}\right)^{m} \rightarrow 1 \\
\quad(\text { as } m \rightarrow \infty) .
\end{array}
$$

Let $\omega_{1}$ and $\omega_{2}$ denote the joint variables

$$
\left(\beta(\tau, \omega), \tau \leqq u_{k}\right) \text { and }\left(\beta(\tau, \omega)-\beta\left(u_{k}, \omega\right), u_{k} \leqq \tau \leqq v\right)
$$

respectively. Then $\xi_{n_{k}}(t, \omega)$ and $C_{n_{k} k_{+1}}^{i}(\omega)$ are expressible as $\xi_{n_{k}}\left(t, \omega_{1}, \omega_{2}\right)$ and $C_{n_{k} k+1}^{i}\left(\omega_{1}\right)$. By the above procedure by which we have defined $\pi_{m}(t, \omega)$ we have 


$$
\begin{aligned}
\xi_{n_{k}}\left(t, \omega_{1}, \omega_{2}\right)=C_{n_{k} k+1}^{i}\left(\omega_{1}\right) & +\int_{u_{k}}^{t} \widetilde{a}_{n_{k}}^{i}\left(\tau, \xi n_{k}\left(t, \omega_{1}, \omega_{2}\right)\right) d t \\
& +\sum_{j} \int_{u_{k}}^{t} \tilde{b}_{n_{k} j}^{i}\left(\tau, \xi n_{k}\left(\tau, \omega_{1}, \omega_{2}\right)\right) d \beta^{j}(t, \omega) .
\end{aligned}
$$

Since the stochastic integral appearing in the above expression is a $B$-mesurable function of $\tilde{b}_{n_{k} j}^{i}\left(t, \xi_{n_{k}}\left(t, \omega_{1}, \omega_{2}\right)\right)$ and $\omega_{1}$ is independent of $\omega_{2}$, we see, by Fubini's theorem in Lebesgue integral, that

$$
\begin{aligned}
\xi_{n_{k}}\left(t, \omega_{1}^{0}, \omega_{2}\right)=C_{n_{k} k+1}^{i}\left(\omega_{1}^{0}\right) & +\int_{u_{k}}^{t} \tilde{a}_{n_{k}}^{i}\left(\tau, \hat{\Sigma}_{n_{k}}\left(\tau, \omega_{1}^{0}, \omega_{2}\right)\right) d \tau \\
& +\sum_{j} \int_{u_{k}}^{t} \widetilde{b}_{n_{k} j}\left(\tau, \xi_{n_{k}}\left(\tau, \omega_{1}^{0}, \omega_{2}\right)\right) d \beta^{j}(\tau, \omega)
\end{aligned}
$$

for almost all $\omega$ except possibly for $\omega_{1}^{0}$ of $P_{\omega_{1} 1}$-measure $0, P_{\omega_{1}}$ being the probability law of $\omega_{1}$.

$\Omega_{n_{0} n_{1}}^{1} \Omega_{n_{1} n_{2}}^{2} \ldots \Omega_{n_{k-1} n_{p}}^{k}$ and $\widetilde{\Omega}_{n_{k}}$ are expressed in the form:

$$
\left\{\omega ; \omega_{1} \in E_{1}\right\},\left\{\omega ; \pi_{m}(t, \omega) \in V_{n_{k}}, \sup _{u_{k} \leqq t \leqq u_{k+1}}\left\|\xi_{n_{k}}(t, \omega)\right\|<2 / 3\right\},
$$

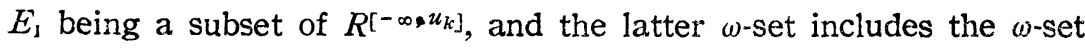

$$
\left\{\omega ; \sup _{u_{k-1} \leqq u_{k}}\left\|\xi_{n_{k}}(t, \omega)-C_{n_{k} k+1}(\omega)\right\|<1 / 3\right\},
$$

as $C_{n_{k} k+1}(\omega) \in S_{1}$. Therefore the left side of (3.16) is greater than

$$
\begin{aligned}
& P_{r}\left\{\omega \in E_{1}, \sup _{u_{k-1} \leqq t \leqq u_{k}}\left\|\xi_{n_{k}}(t, \omega)-C_{n_{k} k+1}(\omega)\right\|<1 / 3\right\} \\
& =P_{r}\left\{\omega_{1} \in E_{1}, \sup _{u_{k-1} \leqq t \leqq u_{k}}\left\|\xi_{n_{k}}\left(t, \omega_{1}, \omega_{2}\right)-C_{n_{k} k+1}\left(\omega_{1}\right)\right\|<1 / 3\right\} \\
& =\int_{E_{1}} P_{r}\left\{\sup _{u_{k-1} \cong t \leqq u_{k}}\left\|\xi \xi_{n_{k}}\left(t, \omega_{1}^{0}, \omega_{2}\right)-C_{n_{k} k+1}\left(\omega_{1}^{0}\right)\right\|<1 / 3\right\} P_{\omega_{1}}\left(d \omega_{1}^{0}\right)
\end{aligned}
$$

(by the independence of $\omega_{1}$ and $\omega_{2}$ )

$$
\begin{aligned}
& \geqslant \int_{E_{1}}\left(1-G(1 / m)^{2}\right) P_{\omega_{1}}\left(d \omega_{1}^{0}\right) \quad \text { (by Theorem 1.3) } \\
& =\left(1-G(1 / m)^{2}\right) P_{r}\left(\omega_{1} \in E_{1}\right),
\end{aligned}
$$

which proves (3.16).

In order to obtain a solution of (3.2) we need only to put

$$
\pi(t, \omega)=\left\{\begin{array}{l}
\pi_{1}(t, \omega) \text { for } \omega \in \Omega^{(\mathbf{i})} \\
\pi_{i}(t, \omega) \text { for } \omega \in \Omega^{(m)}-\Omega^{(m-1)}, \quad m=2,3, \ldots
\end{array}\right.
$$

As to the uniqueness, by making use of the uniqueness of the solution of (2.3), we can easily prove that any solution of (3.2) is coincident with the solution above obtained, which completes the proof of our theorem.

Example 1. If $M$ is a closed manifold, the condition (3.8) follows from (3.10) and so we can do without this condition (3.8). 
Example 2. Let $M$ be a Lie group. If $a^{i}(t, x)$ and $b_{j}{ }^{i}(t, x)$ are left (or right)invariant with regard to the group operation, then the condition (3.8) follows from (3.10). In considering this fact we can define Brownian motions on the Lie group.

A relation between the process we have defined above by the stochastic differential equation (3.2) and the Fokker-Planck equation is given by the following theorem.

THEOREM 3.2. The solution of (3.2) is a simple Markoff process on $M$ and the transition probability law $F(t, p, s, E)$ - the conditional probability law of $\pi(s, \omega)$ under the condition that $\pi(t, \omega)=p$ - is coincident with the probability law of the solution $\pi(s, \omega)(s \geqq t)$ of (3.2) with the initial condition:

$$
\pi(t, \omega) \equiv p
$$

If $f(p)$ is a bounded function of $C_{2}$-class defined on $M$, then we have, as $s \rightarrow t$,

$$
\begin{aligned}
& \frac{1}{s-t}\left\{\int_{x} f(q) F(t, p, s, d q)-f(p)\right\} \\
& \quad \rightarrow\left(\sum_{i} a^{i}(t, x) \frac{\partial}{\partial x^{i}}+\frac{1}{2} \sum_{i j k} b_{k}^{i}(t, x) b_{k}^{i}(t, x) \frac{\partial^{2}}{\partial x^{i} \partial x^{j}}\right) f(x),
\end{aligned}
$$

$x$ being a local coordinate of $p$.

Proof. The first part can be easily proved in the same way as the proof of (3.16). We shall prove the second part. We fix a local coordinate $x$ around $p$ for which $a^{i}(t, x), b_{j}{ }^{i}(t, x)$ are all bounded and we denote by $U$ the coordinate neighbourhood of $x$. We shall denote the solution of (3.2) with the initial condition: $\pi(t, \omega)=p$ by the same notation $\pi(t, \omega)$ and the $x$-coordinate of $\pi(t, \omega)$ with $\xi(t, \omega)$, where we define $\xi(t, \omega)=0$ if $\pi(t, \omega) \notin U$.

Whenever $\pi(s, \omega)$ lies in $U$, we have

$$
\xi^{i}(s, \omega)=x^{i}+\int_{t}^{s} a^{i}(\tau, \xi(\tau, \omega)) d \tau+\sum_{j} \int_{t}^{s} b_{j}^{i}(\tau, \xi(\tau, \omega)) d \beta^{j}(\tau, \omega)
$$

and so, by Theorem 1.1,

$$
\begin{gathered}
f(\xi(s, \omega))-f(x)=\int_{t}^{s}\left\{\sum_{i} a^{i}(\tau, \xi(\tau, \omega)) f_{i}(\xi(\tau, \omega))\right. \\
\left.\quad+\frac{1}{2} \sum_{i j k} b_{k}{ }^{i}(\tau, \xi(\tau, \omega)) b_{k}{ }^{j}(\tau, \xi(\tau, \omega))\right\} d \tau \\
+\sum_{j} \int_{t}^{s} \sum_{i} b_{j}{ }^{i}(\tau, \xi(\tau, \omega)) f_{i}(\xi(\tau, \omega)) d \beta^{j}(\tau, \omega) .
\end{gathered}
$$

In making use of Theorem 1.3 we can easily see that

$$
P_{r}\{\pi(\tau, \omega) \notin U \text { for some } \tau, t \leqq \tau \leqq s\}=O(s-t)^{2} .
$$

Thus we see that $f(\pi(s, \omega))-f(p)$ coincides with the right side of (3.20) ex- 
cept possibly on an $\omega$-set of $P$-measure $O(s-t)^{2}$. Since $f(p)$ is bounded on $M$ by the assumption, we have

$$
\begin{aligned}
& \frac{1}{s-t}\left[\int_{M} f(q) F(t, p, s, d q)-f(p)\right]=\frac{E(f(\pi(s, \omega))-f(p))}{s-t} \\
& =\frac{1}{s-t}\left[\int _ { t } ^ { s } E \left\{\sum_{i} a^{i}(\tau, \xi(\tau, \omega)) f_{i}(\xi(\tau, \omega))\right.\right. \\
& \left.\left.\left.+\frac{1}{2} \sum_{i j k} b_{k}^{i}(\tau, \xi(\tau, \omega)) b_{k}^{j}(\tau, \omega)\right) f_{i j}(\xi(\tau, \omega))\right\} d \tau+O(s-t)^{2}\right] \\
& \rightarrow \sum_{i} a^{i}(t, x) \frac{\partial f}{\partial x^{i}}(x)+\frac{1}{2} \sum_{i j k} b_{k}^{i}(t, x) b_{k}^{j}(t, x) \frac{\partial^{2} f}{\partial x^{i} \partial x^{j}}(x) \quad(s \rightarrow t),
\end{aligned}
$$

which proves (3.18).

\section{REFERENCE}

J. L. Doob:

[1] Stochastic processes depending on a continuous parameter, Trans. Amer. Math. Soc。 42, 1937.

K. Itô:

[1] Stochastic integral, Proc. Imp. Acad. Tokyo, Vol. 20, No. 8 (1944).

[2] On a stochastic integral equation, Proc. Imp. Acad. Tokyo, Vol. 22, No. 2 (1946).

[3] Stochastic differential equations, forthcoming in the Memoirs of Amer. Math. Soc.

A. Kolmogoroff:

[1] Zur Theorie der stetigen zufälligen Prozesse, Math. Ann. 108 (1933)。

[2] Umkehrbarkeit der stetigen Naturgestze, Math. Ann. 113 (1937).

P. Lévy:

[1] Theorie de l'addition des variables aléatoires, Paris (1937).

[2] Processus stochastiques et mouvement brownien, Paris (1948).

F. Perrin:

[1] Étude mathematique du mouvement brownien de rotation, Ann. Éc. Norm., (3), 45 (1928).

K. Yosida:

[1] Brownian motion on the surface of the 3-sphere, Ann. of Math. Statistics, 20, 2 (1949).

[2] Integration of Fokker-Planck's equation in a compact Riemannian space, Arkiv för Mathematik, 1, 9 (1949).

\section{Mathematical Institute, Nagoya University}

